\title{
Advances in the treatment of relapsed/refractory chronic lymphocytic leukemia
}

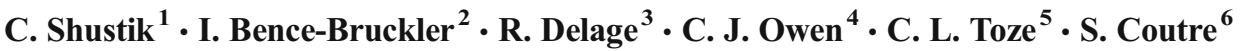

Received: 21 September 2016 / Accepted: 21 March 2017 / Published online: 7 April 2017

(C) The Author(s) 2017. This article is published with open access at Springerlink.com

\begin{abstract}
Treatment of chronic lymphocytic leukemia (CLL) has advanced with the introduction of chemoimmunotherapy (CIT) agents that have improved the outcomes of frontline therapy. However, most treated patients will relapse and require subsequent therapy. This review focuses on recent advances in the treatment of relapsed or refractory CLL. Until recently, treatment options for relapsed CLL were of limited efficacy. Retreatment with fludarabine, cyclophosphamide, and rituximab (FCR) was recommended for patients with a durable response to first-line FCR, although acquired genetic aberrations, impaired marrow reserve, and comorbidities often made this suboptimal therapy for many patients. New options include two agents targeting B cell receptor (BCR) signaling pathways (ibrutinib and idelalisib) and a B cell lymphoma-2 (BCL-2) inhibitor (venetoclax). Allogeneic hematopoietic stem cell transplantation
\end{abstract}

C. Shustik

chaim.shustik@mcgill.ca

McGill University Health Centre, Montreal, Quebec, Canada

2 Department of Hematology, The Ottawa Hospital, Ottawa, ON, Canada

3 Department of Hematology, CHU de Québec-Université Laval, Quebec City, Quebec, Canada

4 Division of Hematology and Hematological Malignancies, University of Calgary, Calgary, AB, Canada

5 Leukemia/BMT Program of BC, Division of Hematology, Vancouver General Hospital, BC Cancer Agency and The University of British Columbia, Vancouver, BC, Canada

6 Division of Hematology, Stanford University School of Medicine, Stanford, CA, USA
(HSCT) remains a potentially curative option for younger patients with a suitable donor.

Keywords CLL $\cdot$ HSCT $\cdot$ Ibrutinib · Idelalisib · Venetoclax

\section{Introduction}

Recent progress in the treatment of chronic lymphocytic leukemia (CLL) has been dramatic with the introduction of several novel agents. The addition of an anti-CD20 monoclonal antibody to frontline chemotherapy (chemoimmunotherapy [CIT]) results in improved progression-free survival (PFS) and overall survival (OS) compared to chemotherapy alone $[1,2]$. However, even with excellent responses to frontline therapy, most patients will relapse with the need for subsequent treatment. The focus of this review will be on recent advances in the treatment of relapsed or refractory CLL.

Chronic lymphocytic leukemia is the most common lymphoproliferative disorder in adults, with an ageadjusted incidence of 4.8 to 5.0 per 100,000 personyears $[3,4]$ and a median age at diagnosis of 72 years [5]. Staging systems using clinical (adenopathy/ organomegaly) and hematologic (anemia and thrombocytopenia) parameters remain useful in stratifying patients in this disease, which has a variable clinical course and a survival that may range from 18 months to more than 20 years $[5,6]$.

Although distinguished by a diagnostic immunophenotype, CLL has been characterized as a heterogeneous disease by genetic and molecular studies. These have identified useful prognostic and predictive variables correlating with the tempo of disease progression and survival, as well as response to therapy. Cytogenetic testing by fluorescence in situ hybridization (FISH) can detect several recurrent genetic aberrations in 
CLL with the most frequent abnormalities at diagnosis being $13 \mathrm{q}$ deletion (55\% of patients), which is associated with a favorable prognosis; $11 \mathrm{q}$ deletion $(18 \%)$ and $17 \mathrm{p}$ deletion (7\% in previously untreated patients and about $30 \%$ in relapsed/refractory patients), both associated with a more rapidly progressive course and shorter survival; and trisomy 12 (16\%), associated with an intermediate prognosis [7]. DNA studies of immunoglobulin genes in CLL have also defined two subsets ofCLL with mutated or unmutated immunoglobulin heavy-chain variable $(I G H V)$ regions based on sequence homology with germ line $I G H V$ genes. Patients with unmutated $I G H V$ have a shorter time to first treatment and PFS following CIT than patients with mutated IGHV. Zeta chain-associated protein kinase $70 \mathrm{kD}$ (ZAP-70) impacts B cell receptor (BCR) signaling, proliferation, and migration and is predominantly expressed in the unmutated genotype, although it is not a reliable surrogate for unmutated $I G H V$ status. Genomic studies have identified mutations involving notch homolog 1 (NOTCH1) and splicing factor $3 \mathrm{~b}$, subunit 1 (SF3B1), which also appear to predict a shorter time to treatment failure and reduced OS [8]. Telomere length has been shown to be a robust independent predictor ofCLL outcomes, including OS and Richter's transformation (transformation into a more aggressive large B cell lymphoma) [9]. An International Prognostic Index for CLL (CLL-IPI) incorporating five independent prognostic features (TP53 status, age, clinical stage, $I G H V$ mutational status, and $\beta 2$-microglobulin level) has recently been developed to allow more targeted CLL patientmanagementinclinical practiceand clinical trials [10].Using a weighted grading of these factors, itidentifies the following four risk groups with significantly different OS at 5 years: low $(93.2 \%)$, intermediate $(79.3 \%)$, high (63.3\%), and very high $(23.3 \%)$ risk.

\section{Currently used therapies}

Treatment of CLL is often deferred in asymptomatic, earlystage patients and initiated in the presence of signs or symptoms outlined by the International Workshop on CLL (IWCLL) criteria [11]. The choice of frontline CLL therapy is influenced by patient age and fitness. Aside from Eastern Cooperative Oncology Group (ECOG) performance status, an approach to formal evaluation of the latter has been the Cumulative Illness Rating Scale (CIRS), which rates comorbidities that may affect tolerability and toxicity of different regimens. With a wider range of therapeutic options now available, categorization of patients based on age $(\geq$ or $<65$, 70 , or 75 years), creatinine clearance $(<$ or $\geq 70 \mathrm{~mL} / \mathrm{min})$, and fitness assessment has become important in the choice of therapy. Improvement in PFS and OS are common goals of therapy, but the risk/benefit ratio of different regimens may be distinct for different risk groups. In younger patients who are better able to tolerate more myelosuppressive regimens, a more intensive approach may be justified, whereas the primary objective in an elderly population may be quality of life with less focus on OS. In chemoimmunotherapy trials, complete response (by IWCLL criteria [11]) and achievement of minimal residual disease (MRD) status $\left(<10^{-4}\right.$ CLL cells detected by multiparameter flow cytometry) are correlated with PFS and OS prolongation. Although MRD negativity may be a clinically important endpoint in potentially curative strategies and in the design of maintenance therapy trials, MRD analysis is not routine in the current standard of care and remains investigational.

Randomized trials have established the combination of fludarabine, cyclophosphamide, and rituximab (FCR) as the frontline standard of care in a younger population with few comorbidities [1, 12]. A 10-year follow-up of 300 patients treated with FCR at MD Anderson Cancer Center indicated a sustained PFS in a subset of patients, with 42 patients experiencing no relapses beyond 10.4 years [13]. The best results were noted in patients lacking poor-risk FISH aberrations and with mutated $I G H V$. Older age ( $\geq 70$ years) was associated with a lower rate of complete remission (CR; 51 vs. $76 \%$ in younger patients), and $54 \%$ of older patients did not complete the planned 6 cycles of therapy due to persistent cytopenias [14]. Based on a phase 2 study of bendamustine plus rituximab (BR) [15], the German CLL group conducted a phase 3 study comparing FCR to BR in previously untreated fit patients (CIRS score $\leq 6$, creatinine clearance $\geq 70 \mathrm{~mL} / \mathrm{min}$ ) [16]. Although treatment with FCR was superior to BR overall for PFS (median 55.2 vs. 41.7 months; $p=0.0003$, hazard ratio [HR] 1.643), no difference in overall survival was observed with a median follow-up of 37.1 months. Furthermore, hematologic toxicity and infectious complications were more pronounced with FCR in patients $>65$ years, and with concerns about protracted immunosuppression with FCR, the combination of bendamustine plus rituximab can be considered an acceptable treatment option for fit patients in this age group. A phase 3 trial in untreated CLL patients with a CIRS score $>6$ or a creatinine clearance of 30 to $60 \mathrm{~mL} / \mathrm{min}$ randomized 781 patients with a median age of 73 years to treatment with chlorambucil (CLB), chlorambucil with rituximab, or chlorambucil with obinutuzumab, a humanized glycoengineered type 2 anti-CD20 MoAb. The latter combination resulted in improved OS compared to CLB and superior PFS and CR rate compared to CLB-rituximab [17] and currently defines the standard of care for this population of patients. Similarly, a phase 3 study comparing the combination of ofatumumab, an anti-CD20 that binds to a different epitope, and chlorambucil to chlorambucil alone confirmed the benefit of the combination in improving PFS in this population (22.4 vs. 13.1 months; $p<0.001)$ [18].

The decision to initiate therapy for relapsed CLL is based on the same considerations as for frontline treatment, although 
observation (i.e., "watch and wait") in patients with slowly progressive lymphocytosis as the only disease manifestation may require closer monitoring than in untreated patients. Until the recent development of kinase inhibitors targeting B cell signaling pathways, treatment options in this population were of limited efficacy. The benefit of adding rituximab to FC in previously treated patients was shown in the REACH trial comparing FCR to FC with improved PFS in the CIT arm (median 30.6 vs. 20.6 months) [2]. Comparable results were observed in the COMPLEMENT 2 trial with the addition of ofatumumab to FC (median 28.9 vs. 18.8 months) [19]. These combinations might be considered for appropriate patients with limited prior therapies, and retreatment with FCR may be effective in patients with a durable response to frontline FCR (progression-free interval exceeding 24-36 months); however, bone marrow suppression is frequent and the duration of a second response is predictably shorter. Further complicating treatment in this setting is the observation of del (17p) and TP53 mutation (by sequence analysis) in $~ 30 \%$ of relapsed patients post-FCR $[20,21]$, which predicts poor response to retreatment with purine nucleosides and alkylating agents. Impaired marrow reserve resulting from previous chemotherapy and additional comorbidities as a consequence of progression of disease and advancing age must also be considered in this setting.

In the minority of patients who are young and fit enough to be eligible, allogeneic hematopoietic stem cell transplantation (allo HSCT), harnessing a "graft vs. leukemia" effect, offers the best chance of cure.

\section{Allogeneic stem cell transplantation}

For selected patients with high-risk CLL and adequate organ function as well as a suitable donor, allo HSCT may be the best option for prolonged survival and possible cure. The potential for long-term disease-free progression (OS 41-65\% at
4-5 years [22-27]; see Table 1) must be balanced against the significant risk of chronic graft-versus-host disease (cGVHD; $44-65 \%$ ) with associated morbidity and the risk of treatmentrelated mortality (TRM). This risk/benefit analysis is based on factors related to disease, patient, and donor [28]. Chronic lymphocytic leukemia with poor initial response to a purine analog-based regimen ( $<$ PR or relapse within 12 months from response) or progression within 24 to 36 months of CIT (FCR, $\mathrm{BR}$, or other anti-CD20-based regimen) identifies high-risk patients [29]. However, the most recent American Society for Blood and Marrow Transplantation (ASBMT) guidelines no longer recommend considering these patients for allograft evaluation in the absence of high-risk FISH mutations (17p deletion, TP53 mutation, or 11q deletion) [30]. Instead, novel agent therapy is proposed for these patients representing a change from previous European Blood and Marrow Transplant guidelines. Patients relapsing who have evidence of clonal evolution and/or complex karyotype, or with del (11q) with suboptimal response or del (17p), should be evaluated for transplant [31]. Novel agents are recommended first in this setting, but emerging data suggest shorter durations of response in patients with del (17p) or complex karyotypes and limited salvage options after failure of novel agents such that allo HSCT should be entertained ideally prior to loss of disease control by novel agents.

Patient selection for allo HSCT is also based on careful consideration of comorbidities that affect TRM and availability of a suitable donor (ideally either a matched sibling or fully matched unrelated donor). The use of reduced-intensity conditioning (RIC) regimens with lower TRM may extend the age eligibility for suitable patients. The integration of novel agents into the pre- or post-transplant setting of allo HSCT is also under investigation. Published data have suggested that ibrutinib may be used safely both pre- and post-allo HSCT [32]. While phase 3 data are lacking, the benefits of allo HSCT in high-risk CLL patients in prolonging PFS have been shown

Table 1 Allogeneic stem cell transplantation in relapsed CLL

\begin{tabular}{|c|c|c|c|c|c|c|}
\hline & $\begin{array}{l}\text { Dreger } 2010 \\
\text { [22] }\end{array}$ & $\begin{array}{l}\text { Khouri } 2011 \\
{[23]}\end{array}$ & $\begin{array}{l}\text { Sorror } 2010 \\
{[24]}\end{array}$ & $\begin{array}{l}\text { Brown } 2012 \\
{[25]}\end{array}$ & Toze 2012 [26] & $\begin{array}{l}\text { Hebenstreit } 2014 \\
{[27]}\end{array}$ \\
\hline$N$ & 90 & 86 & 136 & 76 & 49 & 50 \\
\hline $\begin{array}{l}\text { Median age } \\
\quad \text { (years) }\end{array}$ & 53 & 58 & 56 & 55 & 54 & 58 \\
\hline PFS & $42 \%$ at 4 years & $36 \%$ at 5 years & $32 \%$ at 5 years & $43 \%$ at 5 years & $49 \%$ at 5 years & $63 \%$ at 4 years \\
\hline OS & $65 \%$ at 4 years & $51 \%$ at 5 years & $41 \%$ at 5 years & $63 \%$ at 5 years & $55 \%$ at $5+$ years & $51 \%$ at 4 years \\
\hline Relapse & $40 \%$ at 4 years & $39 \%$ at 3 years & $36 \%$ at 5 years & $40 \%$ at 5 years & $\begin{array}{l}16 \% \text { at } 5 \text { years and } 22 \% \text { at } \\
15 \text { years }\end{array}$ & $37 \%$ at 4 years \\
\hline $\begin{array}{l}\text { Extensive } \\
\text { cGVHD }\end{array}$ & $55 \%$ at 2 years & $56 \%$ at 5 years & $51 \%$ & $65 \%$ at 2 years & $57 \%$ at 5 years & $44 \%$ \\
\hline NRM & $23 \%$ at 4 years & $17 \%$ at 1 year & $32 \%$ at 5 years & $16 \%$ at 5 years & $36 \%$ at 10 years & $30 \%$ at 4 years \\
\hline
\end{tabular}

Predictors for PFS and OS were CR and absence of bulky disease (lymph nodes $>5 \mathrm{~cm}$ )

$c G V H D$ chronic graft-versus-host disease, $N R M$ nonrelapse mortality 
in several studies (Table 1). However, these studies were completed before the use of novel agents, and it is not known if these results will hold in the current setting.

The ASBMT guidelines recommend allo HSCT (a) for standard-risk CLL in the absence of response or if there is evidence of disease progression after BCR inhibitors, (b) for high-risk CLL after failing two lines of therapy and showing an objective response to BCR inhibitors or to a clinical trial or for patients who fail to show an objective response or progress after BCR inhibitors and receive B cell lymphoma-2 (BCL-2) inhibitors regardless of whether an objective response is achieved, and (c) for Richter's transformation upon demonstration of an objective response to anthracycline-based chemotherapy [30]. A RIC regimen is recommended as appropriate.

\section{Newly approved agents}

\section{Ibrutinib}

The molecule ibrutinib was developed as an oral irreversible inhibitor of BTK, an intracytoplasmic enzyme in the BCR signaling pathway that is required for BCR activation of integrins and other molecules. Congenital mutations or absence of BTK in humans leads to profound deficiency of B lymphocytes due to arrest in B cell development with consequent agammaglobulinemia. In CLL, BCR signaling is aberrantly activated, promoting $\mathrm{B}$ cell proliferation and survival as well as modulating migration and homing of malignant cells. The anti-tumor activity of ibrutinib (Table 2) results from disruption of BCR signaling as well as targeting of toll-like receptor signaling and adhesion and migration pathways.

An initial phase $1 b / 2$ clinical trial in relapsed/refractory CLL [20] studied the safety, efficacy, and pharmacokinetics of ibrutinib. Eighty-five patients with a median of 4 (1-12) prior therapies, almost all exposed to a purine nucleoside and rituximab, were treated with ibrutinib at a dose of $420 \mathrm{mg}$ $(n=51)$ or $840 \mathrm{mg}(n=34)$. Deletion $17 \mathrm{p}$ was present in $33 \%$ and del (11q) in 36\%. The overall response rate (ORR) was $71 \%$ at both dose levels, and responses were independent of adverse cytogenetics. At 26 months, PFS for the entire cohort was $75 \%$ and OS $83 \%$. The responses were predominantly partial with the observation of an early transient increase in lymphocytes, with frequent persistent peripheral lymphocytosis, despite regression of adenopathy and splenomegaly and improvement in other hematologic values. This phenomenon of an early rise in lymphocyte count has been attributed to dislodging of CLL cells from nodal compartments into the circulation. The recognition that persistent or increased lymphocytosis is not indicative of treatment failure with these agents has necessitated a revision of response criteria with the addition of partial response with lymphocytosis $\left(\mathrm{PR}_{\mathrm{L}}\right)$ [33]. Based on the results of these early phase studies, ibrutinib received FDA-accelerated approval in relapsed CLL in 2014 [34], then was accepted as a breakthrough drug for CLL with $17 \mathrm{p}$ deletion the same year [35]. This study established the 420-mg daily dose for subsequent trials with identical BTK occupancy at 96 to $99 \%$ for both the 420- and 840-mg doses [36].

The efficacy of ibrutinib was confirmed in the RESONATE $^{\mathrm{TM}}$ trial, a phase 3 comparison of ibrutinib to ofatumumab in patients with relapsed or refractory CLL with PFS as the primary endpoint [37]. Eligibility criteria included at least one prior therapy and ineligibility for purine analog treatment due to comorbidities, age over 70 years, presence of del (17p), or short duration of response after CIT. In this multicenter study, 391 patients were randomly allocated to receive ibrutinib $420 \mathrm{mg}$ daily until disease progression $(n=195)$ or ofatumumab at an initial dose of $300 \mathrm{mg}$, followed by $2000 \mathrm{mg}$ weekly for 7 weeks, then every 4 weeks for 16 weeks ( $n=196)$. The baseline characteristics were well balanced with a median of $3(1-12)$ prior therapies in the ibrutinib group and $2(1-13)$ in the ofatumumab group; del (17p) and del

Table 2 Trials involving new agents

\begin{tabular}{|c|c|c|c|c|c|c|c|}
\hline Drug & Phase & Number & $\begin{array}{l}\text { Prior lines of } \\
\text { therapy (median) }\end{array}$ & ORR & $\mathrm{CR}$ & PFS & $p$ Value \\
\hline Ibrutinib [20] & $1 b / 2$ & 85 & 4 & $71 \%$ & $2 \%$ & $75 \%$ at 26 months & $\mathrm{n} / \mathrm{a}$ \\
\hline Ibrutinib vs. ofatumumab [37] & 3 & 391 & 3 & 42.6 vs. $4.1 \%$ & 2 vs. $1 \%$ & Not reached vs. 8.1 months & $p<0.001$ for $\mathrm{ORR}$ \\
\hline Ibrutinib [38] & $1 \mathrm{~b} / 2$ & $101^{\mathrm{a}}$ & 4 & $90 \%$ & $7 \%$ & $69 \%$ at 30 months & $\mathrm{n} / \mathrm{a}$ \\
\hline $\begin{array}{l}\text { Idelalisib + rituximab vs. } \\
\text { placebo + rituximab [52] }\end{array}$ & 3 & 220 & $\begin{array}{c}\text { Anti-CD20-based } \\
\text { or } \geq 2 \text { previous }\end{array}$ & 81 vs. $13 \%$ & $0 \%$ & Not reached vs. 5.5 months & $p<0.001$ for $\mathrm{ORR}$ \\
\hline Venetoclax $[60]$ & 1 & 56 & 4 & $84 \%$ & $21 \% \mathrm{CR} / \mathrm{Cr}_{\mathrm{i}}$ & $\mathrm{n} / \mathrm{a}$ & $\mathrm{n} / \mathrm{a}$ \\
\hline Venetoclax [61] & 1 & 116 & 3 & $79 \%$ & $20 \%$ & 25 months & $\mathrm{n} / \mathrm{a}$ \\
\hline Venetoclax [63] & 2 & 107 & 2 & $79 \%$ & $8 \%$ & Not reached at 12.1 months & $\mathrm{n} / \mathrm{a}$ \\
\hline Venetoclax + rituximab [62] & 1 & 49 & 2 & $84 \%$ & $41 \% \mathrm{CR} / \mathrm{Cr}_{\mathrm{i}}$ & $\mathrm{n} / \mathrm{a}$ & $\mathrm{n} / \mathrm{a}$ \\
\hline
\end{tabular}

${ }^{\mathrm{a}}$ Relapsed/refractory CLL patients only 
(11q) were each detected in about $30 \%$ of patients in both arms. The study was terminated after a pre-planned interim analysis demonstrated markedly improved outcomes for the ibrutinib arm. With a median follow-up of 9.4 months, median PFS in the ofatumumab arm was 8.1 months and had not been reached in the ibrutinib arm with a HR for progression or death in the ibrutinib arm of $0.22(p<0.001)$. A crossover design permitted patients progressing on ofatumumab to receive ibrutinib once the primary endpoint was reached, and at the time of analysis, 57 patients had crossed over to ibrutinib. Nonetheless, an OS advantage for the ibrutinib therapy was observed in both uncensored and censored for crossover groups (HR for death in ibrutinib arm 0.39 and 0.43 , respectively, at 12 months; OS $90 \%$ in ibrutinib group and $81 \%$ in ofatumumab group). Improvement in PFS was observed across all subgroups regardless of age, clinical stage, IGHV mutation status, or presence of del (17p).

An update of the initial phase $1 \mathrm{~b} / 2$ trial reported 3-year follow-up of 31 treatment-naïve (TN) patients and 101 patients with relapsed/refractory CLL treated with single-agent ibrutinib [38]. At a median of 30 and 23 months on study for $\mathrm{TN}$ and relapsed/refractory patients, 81 and $53 \%$, respectively, remained on drug. Response quality improved with time; with extended follow-up, 94\% of patients who achieved $\mathrm{PR}_{\mathrm{L}}$ converted to CR or PR. Discontinuation due to disease progression was only $3 \%$ in the TN group but $21 \%$ in the relapsed/ refractory group, whereas discontinuation due to drug intolerance was similar in both groups (10 and 12\%, respectively). The estimated PFS at 30 months was 96 and $69 \%$ for the two groups but only $48 \%$ in patients with del (17p).

The toxicities observed with ibrutinib are modest, with the majority of reported adverse events (AEs) being grade 1-2. The most frequent nonhematologic AEs occurring in at least $20 \%$ of patients were diarrhea, bleeding, fatigue, pyrexia, and nausea. In the RESONATE study, AEs of grade 3 or higher in the ibrutinib arm included atrial fibrillation (AF) in $3 \%$, although subsequent reports noted increasing AF prevalence with additional time on ibrutinib [39]. Another study found that about $6 \%$ of all newly diagnosed CLL patients had a history of AF; in those without such a history, the background CLL population incidence of AF was about $1 \%$ per year [40]. A recent systematic review and meta-analysis found that the pooled relative risk of AF associated with ibrutinib as compared to the comparator in randomized trials was 3.5 to 3.9, depending on the model used. The pooled rate of AF among ibrutinib recipients from all trials examined was 3.3 per 100 person-years [41]. Arrhythmic symptoms or new-onset dyspnea in patients receiving ibrutinib should be evaluated clinically, with electrocardiography if appropriate. Ibrutinib therapy should be withheld in patients with new-onset or worsening grade 3 or 4 toxicities and reinitiated at the starting dose once symptoms have resolved [42]. In the RESONATE, RESONATE-2, and HELIOS trials, most patients with AF were able to continue ibrutinib treatment and did not discontinue due to AF [39, 43, 44].

Bleeding-related AEs, most commonly petechiae or ecchymoses, have also been reported with ibrutinib (44\% with ibrutinib vs. $12 \%$ with ofatumumab in RESONATE), but major hemorrhage (grade 3 or higher or requiring red cell transfusion or hospitalization) occurred in only two patients in the ibrutinib group and three in the ofatumumab group. A study of single-agent ibrutinib in CLL found that the cumulative incidence of grade $\leq 2$ bleeding-related AEs plateaued by 6 months, suggesting that the risk of bleeding decreases with continued therapy [45]. Ibrutinib should be withheld for at least 3 to 7 days pre- and post-surgery depending on the type of surgery and the risk of bleeding, and vitamin $\mathrm{K}$ antagonists should not be administered concomitantly. If therapeutic anticoagulation is required, consider temporarily withholding ibrutinib until stable anti-coagulation is achieved [42].

As impaired humoral immunity and increased infection risk resulting from panhypogammaglobulinemia are characteristic of advanced CLL, the effect of BTK inhibition on normal $\mathrm{B}$ cell function in CLL may have clinical relevance. A study of 86 patients with previously untreated or relapsed/ refractory CLL receiving ibrutinib for at least 12 months [46] found a progressive decline in serum immunoglobulin $G$ (IgG) levels, while immunoglobulin A (IgA) levels increased with treatment. In patients with a $\geq 50 \%$ increase in IgA level, the infection rate was decreased, suggesting partial immune recovery with ibrutinib therapy. Patients taking ibrutinib in RESONATE also experienced increased IgA levels, as well as sustained improvements in hemoglobin, platelet levels, and absolute neutrophil count (ANC) compared with patients taking ofatumumab [47]. Another study found low rates of treatment-emergent autoimmune cytopenias (AICs) with ibrutinib treatment, and 19 of 22 patients receiving corticosteroids for autoimmune hemolytic anemia at the start of ibrutinib therapy were able to discontinue them with resolution of the hemolytic process [48].

\section{Idelalisib}

Idelalisib (Table 2) is an orally bioavailable inhibitor of the delta isoform of PI3K, the predominant PI3K isoform in $\mathrm{B}$ cells. PI3K has limited expression in other hematopoietic cells, and thus, PI3K inhibition acts as a targeted B cell therapy. As an inhibitor of PI3K signaling downstream from the BCR in CLL cells, this drug also interrupts BCR signaling pathways. However, idelalisib may also disrupt the protective effect of the CLL microenvironment [49] by interfering with chemokine networks, including CXCR4, CD40, and CD49d effects on multiple signaling pathways [50]. It was approved by the FDA in 2014 for the treatment of relapsed CLL in combination with rituximab [51]. 
In phase 1 studies, idelalisib was investigated as a single agent and in combination with many other chemoimmunotherapeutic agents in relapsed or refractory CLL patients. The clinical activity and acceptable toxicity led to a pivotal phase 3 randomized trial of idelalisib plus rituximab vs. rituximab plus placebo [52]. Patients were eligible if they had progressed within 24 months of their last treatment (which must have included an anti-CD20-based therapy or at least two prior cytotoxic regimens) and were not candidates for cytotoxic drugs due to impaired marrow reserve as a consequence of prior myelosuppressive therapy, or a creatinine clearance $<60 \mathrm{~mL} / \mathrm{min}$, or a CIRS score $>6$. Of the patients, 222 were allocated to treatment with rituximab $375 \mathrm{mg} / \mathrm{m}^{2}$ as an initial dose, followed by $500 \mathrm{mg} / \mathrm{m}^{2}$ every 2 weeks for four doses then every 4 weeks for three doses (for a total of eight infusions) in combination with either idelalisib $150 \mathrm{mg}$ or placebo twice daily. Patients (median age 71 years) were stratified by $I G H V$ mutation status and the presence of del (17p) or TP53 mutation (present in 40\%). Baseline characteristics, including hematologic values, CIRS scores, and number and type of prior therapies, were well balanced. At 24 weeks, $93 \%$ of patients in the rituximab-idelalisib group were progression-free compared to $46 \%$ in the rituximabplacebo arm, and the study was stopped at this pre-specified point. Median PFS in the rituximab-placebo arm was 5.5 months and had not been reached in the idelalisib with rituximab cohort (HR for progression or death in the idelalisib arm, $0.15 ; 95 \%$ confidence interval 0.08 to $0.28 ; p<0.001$ ). This clinical benefit for the combination was observed in all pre-specified subgroups including high-risk patients with del (17p) and/or TP53 mutation. Updated results of this study [53] reported a median PFS of 16.6 months in the latter group and 20.3 months in patients without either abnormality.

In this study, the most frequently observed grade 3 or higher adverse events attributed to idelalisib were diarrhea (5\%) and increases in hepatic transaminases (8\%) [53]. Adverse events of any grade included neutropenia (60\%), transaminase elevation (40\%), anemia (29\%), thrombocytopenia $(19 \%)$, bleeding (14\%), pneumonia (10\%), rash $(10 \%)$, and pneumonitis $(6 \%)$. Diarrhea/colitis of any grade occurred in $21 \%$ of patients on idelalisib and was managed by drug interruption, corticosteroids, and symptom management. The incidence of diarrhea/colitis is underrepresented in the early publications due to limited time on therapy for most patients at the time of analysis. Later reports with longer follow-up report a higher incidence of diarrhea/colitis, an adverse event that requires careful monitoring and rapid treatment [54]. The distinct late-onset diarrhea occurs with a median onset of more than 6 months of therapy and may be associated with colitis with lymphocytic infiltration on biopsy [55]. This adverse event requires drug interruption and typically requires steroid therapy for rapid resolution. Either prednisone or nonabsorbable corticosteroids (e.g., budesonide) are generally effective in ameliorating this condition. Once the diarrhea resolves, the patient can often be successfully rechallenged with idelalisib and the steroids tapered off. Hepatic enzyme elevations were reversible by withholding drug, and idelalisib could be restarted in most patients without recurrence. Pneumonitis has also been reported with idelalisib in other studies, including fatal cases, without identifiable pathogens and with no defined mechanism. A 2015 consensus paper summarizes experience in the management of these more concerning toxicities of idelalisib [56]. During safety monitoring of several trials of idelalisib in untreated CLL and relapsed low-grade lymphoma, an increased incidence of serious infection was observed and these trials were terminated. Health care professionals were advised of these results by the manufacturer (Gilead Sciences), and prophylaxis for Pneumocystis carinii and monitoring for cytomegalovirus reactivation have consequently been mandated during idelalisib treatment [57].

\section{Venetoclax}

Venetoclax (Table 2), a BH3 mimetic, is an orally administered small molecule that potently inhibits the anti-apoptotic BCL-2 protein with limited effect on $\mathrm{BCLX}_{\mathrm{L}}$, a related antiapoptotic protein important for platelet survival. Venetoclax induces rapid onset apoptosis of CLL cells, apparently independently of TP53 function. Analysis of both in vivo and in vitro results from a phase 1 trial showed that the depth of clinical responses to venetoclax were also independent of chromosome $17 \mathrm{p}$ deletion, TP53 mutation, and TP53 function [58]. This agent has recently been approved by the FDA for the treatment of previously treated CLL with $17 \mathrm{p}$ deletion in the USA [59] and is currently being studied in phase 2 and 3 trials.

In an initial phase 1 trial [60], 56 patients with relapsed/ refractory CLL or small lymphocytic lymphoma (SLL), including 17 with del (17p), were treated with single-agent venetoclax. The ORR was $84 \%$ (82\% in del [17p]) including rates of $20 \% \mathrm{CR}$ or $\mathrm{CR}$ with incomplete marrow recovery $\left(\mathrm{Cr}_{\mathrm{i}}\right)$. Tumor lysis syndrome (TLS) developed in five patients, leading to the suspension of the trial to reassess dosing of the drug. The trial was restarted with a weekly stepwise dosing schedule with no subsequent observed TLS.

Roberts reported the results of a phase 1 study of venetoclax in combination with rituximab in 49 patients with relapsed/refractory CLL [61]. A CR/Cr $\mathrm{r}_{\mathrm{i}}$ rate of $41 \%$ was observed, including MRD negativity in $65 \%$ (13/20) of these patients (49\% [24/49] overall). These responses were sustained in six patients achieving $\mathrm{CR} / \mathrm{CR}_{\mathrm{i}}$ for up to 21 months after discontinuing treatment. The most frequent grade 3 or higher AEs were neutropenia (51\%), thrombocytopenia $(16 \%)$, and anemia (14\%), and there was one death due to TLS. 
The updated results of the initial phase 1 dose-escalation study of daily oral venetoclax, including an expansion cohort of 60 additional patients, have recently been reported [62]. In the dose-escalation phase, 56 patients with relapsed/refractory CLL or SLL received daily venetoclax in doses ranging from 150 to $1200 \mathrm{mg}$. In an expansion cohort of 60 additional patients, venetoclax was escalated in weekly stepwise increments to $400 \mathrm{mg}$ daily. Patients enrolled in this study had received a median of three prior lines of therapy although none with prior ibrutinib or idelalisib. Patients with autoimmune cytopenias were excluded. Pooled ORRs of 71 to $79 \%$ were observed in subgroups with adverse prognostic features including fludarabine resistance, del (17p), and unmutated $I G H V$. Complete remissions were observed in $20 \%$ of the patients in both cohorts, and 5\% had undetectable MRD by flow cytometry. With a median follow-up of 17 months in the cohort treated at $400 \mathrm{mg}$ daily, the median PFS could not be reliably estimated, but the rate of PFS was estimated to be $66 \%$ at 15 months with the likelihood that the CR rate would increase with longer observation. In the expansion cohort following dose-escalation adjustments, no cases of clinical tumor lysis were observed.

A multicenter, phase 2, single-arm study examined venetoclax monotherapy (using a stepped-dose schedule) in relapsed or refractory del (17p) CLL [63]. At a median followup of 12.1 months, an ORR of 79\% was achieved ( 85 of 107 patients), with a $\mathrm{CR} / \mathrm{Cr}_{\mathrm{i}}$ of $8 \%$, a nodular PR of $8 \%$, and a $\mathrm{PR}$ of $69 \%$. MRD in peripheral blood was not detectable in 18 of 45 assessed patients. The most common grade 3-4 adverse events were neutropenia (40\%), infection (20\%), anemia $(18 \%)$, and thrombocytopenia (15\%). Serious adverse events occurred in 55\% of patients, irrespective of their relationship to treatment, with pyrexia, autoimmune hemolytic anemia, pneumonia, and febrile neutropenia seen most commonly. Laboratory TLS was reported in five patients during the ramp-up period (four within the first 2 days of treatment and one at week 3) but resolved without clinical sequelae.

Despite the lack of clinical TLS after the institution of the slow stepwise increase in dose of venetoclax, it remains important to monitor for laboratory abnormalities indicating TLS, particularly in patients considered at higher risk because of a significantly elevated blood lymphocyte count $(>25 \mathrm{Gi} / \mathrm{L})$ or those with bulky adenopathy $(>5 \mathrm{~cm}$ ). In appropriate cases, hospitalization for dose escalations of venetoclax is required.

\section{Discussion}

\section{Patient selection for specific agents}

Despite the improvement in response rates and disease control with frontline chemoimmunotherapy in CLL, disease relapse remains the norm. Although a subset of patients with mutated
$I G H V$ may experience long PFS after CIT with FCR, the majority of patients will eventually require subsequent therapy. With the broader availability of newer agents, the challenge for the clinician is selection and sequencing of these drugs.

Retreatment with FCR may be considered for suitable patients experiencing an initial PFS exceeding 24 to 36 months; however, impaired marrow reserve following this treatment and the emergence of a del (17p) clone may limit the efficacy of this regimen. In addition, there is significant concern about the increased risk of myelodysplasia with repeated exposure to fludarabine. In a minority of younger patients, allo HSCT should be considered a potentially curative approach if a human leukocyte antigen (HLA)-matched donor is available, but for most patients, transplantation will not be feasible due to age and/or comorbidities and many patients will achieve good results with novel agents in this setting.

While disease relapse characterized by slowly progressive lymphocytosis may not require immediate reinstitution of therapy, subsequent treatment decisions will be guided by the same factors determining initial therapy, including patient age and concurrent comorbidities, as well as marrow reserve, which may be impaired as a result of prior treatment. Repeat cytogenetic assessment should be performed since the presence of del (17p) is critical to treatment decisions, and the frequency of this event increases with subsequent relapses. Current options for treatment with novel drugs have been reviewed above. In the absence of evidence from randomized trials directly comparing the agents under discussion, preferences may be determined by patient characteristics. Ibrutinib, idelalisib, and venetoclax are all active in relapsed CLL with del (17p). With respect to depth of response beyond CR, MRD negativity has been associated with ibrutinib in combination (18\% in the HELIOS trial in combination with BR) [64], but rarely with ibrutinib monotherapy. Venetoclax has been associated with MRD responses when given as monotherapy (17\%) [63]. The impact of achieving this degree of response on OS in relapsed CLL remains to be established; ibrutinib has demonstrated conclusive OS benefit in randomized trials without achieving MRD negativity. The ease of administration of oral, once-daily ibrutinib vs. the concomitant requirement for 8 cycles of intravenous rituximab with oral, twicedaily idelalisib may be a consideration in favor of ibrutinib for some patients, as may the option for dose reduction in patients with comorbidities (although dose reductions for this reason are based on physician preferences rather than trial data). Conversely, the need for anti-coagulation therapy or a prior history of atrial fibrillation may favor idelalisib, depending on clinician preference. An indirect comparison of ibrutinib monotherapy and idelalisib plus ofatumumab [65] suggested a longer PFS and fewer discontinuations with ibrutinib, although a head-to-head trial is required for a true comparison. In appropriate patients (those who have achieved a lengthy first remission after CIT), retreatment with CIT remains a 
reasonable option and has the advantage of a short duration of therapy and a subsequent treatment-free interval.

\section{Resistance, progression, and sequencing}

Disease progression occurring in patients after prolonged treatment with ibrutinib has been associated with poor prognosis and short survival (median 17.6 months after CLL progression and 3.5 months if Richter's transformation had occurred) [66]. However, the patients included in this analysis were from early clinical trials with ibrutinib and had largely exhausted standard treatment options when they entered the trials (median of three prior therapies). In an analysis of RESONATE and RESONATE- 2 patients, 23 patients who had discontinued first- or second-line ibrutinib had not yet reached a median OS, compared with a median OS of 79 months in 34 patients who discontinued third-line ibrutinib or beyond [67]. Of the 31 previously untreated patients who received ibrutinib as initial therapy in the phase 1 trial [68], only one has been reported with subsequent ibrutinib failure. Richter's transformation has been reported as an early complication in the course of therapy with ibrutinib, but typically in previously treated patients with adverse cytogenetic features, including complex karyotype and MYC abnormalities on FISH; these patients may have had early Richter's at study entry. Studies in patients developing resistance to ibrutinib have identified point mutations in the BTK binding site C481S, resulting in loss of BTK inhibition, and in the immediate downstream kinase PLC $\gamma 2$ [69]. A retrospective analysis of 123 CLL patients who discontinued ibrutinib- or idelalisib-based therapy found that many patients who discontinued these therapies due to toxicity or progression responded to other therapies $\left(40 \% \mathrm{PR}+\mathrm{PR}_{\mathrm{L}}\right.$ to nonkinase inhibitors and $60-67 \%$ to other kinase inhibitor therapy) [70]. Preliminary results from an ongoing phase 2 trial also suggest that venetoclax monotherapy is active in CLL patients relapsing after idelalisib or ibrutinib [71].

Lenalidomide, an immunomodulatory drug with potent in vitro activity in CLL, remains under investigation in CLL, but one randomized study comparing it to chlorambucil was terminated early due to excess mortality in the lenalidomide arm [72]. Acalabrutinib, a second-generation BTK inhibitor that does not irreversibly target alternative kinases, has been investigated in a phase 1/2 study [73]. A phase 3 study comparing this drug to ibrutinib in high-risk patients with relapsed CLL has been initiated. Clinical trials of other agents including XPO1 inhibitors (e.g., selinexor), Syk inhibitors (fostamatinib and entospletinib), new BTK inhibitors (BGB311), and new PI3K inhibitors (buparlisib, duvelisib, and TGR-1202) are also in progress. The dramatic responses observed with CAR-T cell therapy in small numbers of advanced, refractory CLL patients suggest the possibility of effective immunotherapeutic strategies in the future.

\section{Combination therapy}

Ibrutinib and idelalisib have each been combined with BR in phase 3 clinical trials comparing the three-drug combination with BR alone. In both cases, the addition of the new agent resulted in significant improvements in outcome [74, 75]. Two-year results for the HELIOS trial, which studied the combination of ibrutinib with BR, found that over a median follow-up of 25.4 months, the triple combination was superior to BR alone in PFS (not reached vs. 14.2 months), 2-year PFS (74.8 vs. $20.9 \%), \mathrm{CR} / \mathrm{Cr}_{\mathrm{i}}(33.9$ vs. $7.2 \%)$, best ORR at any time point (87.2 vs. $66.1 \%$ ), and MRD-negative response

Table 3 Summary of phase 3 trial results in relapsed/refractory CLL

\begin{tabular}{|c|c|c|c|c|c|c|c|}
\hline Trial & Agents & Design & Number & $\begin{array}{l}\text { Median } \\
\text { age }\end{array}$ & Median PFS (months) & $\begin{array}{l}\text { Median follow-up } \\
\text { (months) }\end{array}$ & MRD \\
\hline REACH [2] & FCR vs. FC & Open label & 552 & 62.5 & $\begin{array}{l}30.6 \text { vs. } 20.6 \text { months } \\
\quad(p<0.001)\end{array}$ & 25 & 13 vs. $12 \%$ \\
\hline RESONATE [37] & Ibrutinib vs. ofatumumab & Open label & 391 & 67 & $\begin{array}{l}\text { Not reached vs. } \\
8.1 \text { months }\end{array}$ & 9.4 & $\mathrm{n} / \mathrm{a}$ \\
\hline Furman 2014 [52] & $\begin{array}{l}\text { Idelalisib + rituximab vs. } \\
\text { rituximab }\end{array}$ & Double-blind & 220 & 71 & $\begin{array}{l}\text { Not reached vs. } \\
5.5 \text { months }\end{array}$ & 3.8 and $2.9^{\mathrm{a}}$ & $\mathrm{n} / \mathrm{a}$ \\
\hline HELIOS [74] & Ibrutinib + BR vs. BR & Double-blind & 578 & 63.5 & $\begin{array}{l}\text { Not reached vs. } 13.3 \\
\quad(p<0.0001)\end{array}$ & 17 & $\begin{array}{l}18 \text { vs. } 5 \% \\
\quad(p=0.0011)\end{array}$ \\
\hline $\begin{array}{l}\text { Zelenetz } 2015 \\
\quad[75]\end{array}$ & Idelalisib + BR vs. BR & Double-blind & 416 & $\begin{array}{l}58 \%< \\
\quad 65 \text { years }\end{array}$ & 23 vs. $11(p<0.0001)$ & 12 & $\mathrm{n} / \mathrm{a}$ \\
\hline $\begin{array}{l}\text { COMPLEMENT } \\
2 \text { [19] }\end{array}$ & Ofatumumab + FC vs. FC & Open label & 365 & 61 & $\begin{array}{l}28.9 \text { vs. } 18.8 \\
\qquad(p=0.0032)\end{array}$ & 34 & $\begin{array}{l}21 \text { vs. } 8 \% \\
\quad(p=0.0006)\end{array}$ \\
\hline Jones 2016 [76] & $\begin{array}{l}\text { Idelalisib + ofatumumab vs. } \\
\text { ofatumumab }\end{array}$ & Open label & 261 & 67 & $\begin{array}{l}16.4 \text { vs. } 8.0 \\
\quad(p<0.0001)\end{array}$ & 12.3 & $\mathrm{n} / \mathrm{a}$ \\
\hline
\end{tabular}

${ }^{\mathrm{a}}$ Time receiving study drug 
( 18.0 vs. $4.8 \%$ in the intent-to-treat population). Median OS remained unreached in both treatment arms and there were no new safety findings [64].

The combination of idelalisib and ofatumumab was compared with ofatumumab alone in an open-label phase 3 study in 261 patients with relapsed CLL [76] (Table 3). The combination demonstrated superior median PFS (16.4 vs. 8.0 months; $p<0.0001)$ and ORR (75.3 vs. $18.4 \% ; p<0.0001)$.

The combination of idelalisib and entospletinib was tested in a phase 2 trial and produced an ORR of $60 \%$ in relapsed CLL patients with a median treatment exposure of 10 weeks. However, the study was terminated early due to treatmentemergent pneumonitis in $18 \%$ of patients ( 12 cases, 11 of which were severe), resulting in two fatalities [77].

The combination of ibrutinib and FCR was studied as firstline therapy in young, fit CLL patients in a phase 2 trial. Early results in 17 restaged patients showed an ORR of $100 \%$ after a median of 7.7 months of therapy, 8 patients (47\%) with CR or $\mathrm{CR}_{\mathrm{L}}$, all of whom were MRD-negative, and 9 (53\%) with PR [78].

A number of other combination regimens involving agents discussed above are in ongoing clinical trials in CLL patients. Regimens under investigation include ibrutinib plus obinutuzumab, ibrutinib plus lenalidomide, ibrutinib plus lenalidomide plus rituximab, ibrutinib plus selinexor, and venetoclax plus ibrutinib plus obinutuzumab. The combination of venetoclax and sunitinib may also be worth investigating, based on the results of an in vitro study that found that sunitinib may overcome venetoclax resistance in some patients by downregulating BCL-xl, Mcl-1, and A1 in CLL cells [79].

Acknowledgments This work was financially supported by Janssen Inc., the manufacturer of Imbruvica ${ }^{\circledR}$ (ibrutinib). Medical writing services from CTC Communications were funded by Janssen Inc. Manuscript concept was initiated by Janssen Inc.

\section{Compliance with ethical standards}

Conflicts of interest All authors have completed the International Committee of Medical Journal Editors (ICMJE) uniform disclosure at www.icmje.org/coi_disclosure.pdf and declared financial support for the submitted work from Janssen Canada. C. S. has received honoraria for advisory board memberships from Janssen, AbbVie, and Gilead Sciences; I. B.-B. has received honoraria for advisory board memberships from Janssen, Gilead Sciences, and Lundbeck; R. D. has received grants from Roche, Novartis, Janssen, and Pfizer; C. O. has received honoraria for advisory board membership from AbbVie and other honoraria from Janssen and Gilead Sciences; C. T. received grants and/or personal fees for advisory board memberships from Janssen, Lundbeck, Gilead Sciences, and GSK; and S. C. has received honoraria for advisory board memberships from Pharmacyclics, Gilead, and Janssen.

Statement of human and animal rights research Ethics board approval was obtained from all the three sites in accordance with the ethical standards as laid down in the 1964 Declaration of Helsinki and its later amendments.
Open Access This article is distributed under the terms of the Creative Commons Attribution 4.0 International License (http:// creativecommons.org/licenses/by/4.0/), which permits unrestricted use, distribution, and reproduction in any medium, provided you give appropriate credit to the original author(s) and the source, provide a link to the Creative Commons license, and indicate if changes were made.

\section{References}

1. Hallek M, Fischer K, Fingerle-Rowson G et al (2010) Addition of rituximab to fludarabine and cyclophosphamide in patients with chronic lymphocytic leukaemia: a randomised, open-label, phase 3 trial. Lancet 376:1164-1174

2. Robak T, Dmoszynska A, Solal-Céligny P et al (2010) Rituximab plus fludarabine and cyclophosphamide prolongs progression-free survival compared with fludarabine and cyclophosphamide alone in previously treated chronic lymphocytic leukemia. J Clin Oncol 28: $1756-1765$

3. Howlader N, Noone AM, Krapcho M, et al. (eds). SEER Cancer Statistics Review, 1975-2013, National Cancer Institute. Bethesda, MD, http://seer.cancer.gov/csr/1975_2013/, based on November 2015 SEER data submission, posted to the SEER web site, April 2016.

4. Statistics Canada. Table 1030553: new cases and 1991 agestandardized rate for primary cancer (based on the August 2015 CCR tabulation file), by cancer type and sex, Canada, provinces and territories. Available at: http://www5.statcan.gc.ca/cansim/ a26?lang=eng\&id=1030553. Accessed November 16, 2016.

5. Eichhorst B, Dreyling M, Robak T, Montserrat E, Hallek M, ESMO Guidelines Working Group (2011) Chronic lymphocytic leukemia: ESMO clinical practice guidelines for diagnosis, treatment and follow-up. Ann Oncol 22(Suppl 6):vi50-vi54

6. Siddon AJ, Rinder HM, Education Committee of the Academy of Clinical Laboratory Physicians and Scientists (2013) Pathology consultation on evaluating prognosis in incidental monoclonal lymphocytosis and chronic lymphocytic leukemia. Am J Clin Pathol 139:708-712

7. Döhner H, Stilgenbauer S, Benner A et al (2000) Genomic aberrations and survival in chronic lymphocytic leukemia. N Engl J Med 343:1910-1916

8. Nabhan C, Raca G, Wang YL (2015) Predicting prognosis in chronic lymphocytic leukemia in the contemporary era. JAMA Oncol 1: 965-974

9. Rossi D, Lobetti Bodoni C, Genuardi E et al (2009) Telomere length is an independent predictor of survival, treatment requirement and Richter's syndrome transformation in chronic lymphocytic leukemia. Leukemia 23:1062-1072

10. The International CLL-IPI working group (2016) An international prognostic index for patients with chronic lymphocytic leukaemia (CLL-IPI): a meta-analysis of individual patient data. Lancet Onco 17:779-790

11. Hallek M, Cheson BD, Catovsky D, International Workshop on Chronic Lymphocytic Leukemia et al (2008) Guidelines for the diagnosis and treatment of chronic lymphocytic leukemia: a report from the International Workshop on Chronic Lymphocytic Leukemia updating the National Cancer Institute-Working Group 1996 guidelines. Blood 111:5446-5456

12. Fischer K, Bahlo J, Fink AM et al (2016) Long term remissions after FCR chemoimmunotherapy in previously untreated patients with CLL: updated results of the CLL8 trial. Blood 127:208-215

13. Thompson PA, Tam CS, O'Brien SM et al (2016) Fludarabine, cyclophosphamide and rituximab achieves long-term disease-free 
survival in IGHV-mutated chronic lymphocytic leukemia. Blood 127:303-309

14. Tam CS, O'Brien S, Wierda W et al (2008) Long-term results of the fludarabine, cyclophosphamide, and rituximab regimen as initial therapy of chronic lymphocytic leukemia. Blood 112:975-980

15. Fischer K, Cramer P, Busch R et al (2012) Bendamustine in combination with rituximab for previously untreated patients with chronic lymphocytic leukemia: a multicenter phase II trial of the German Chronic Lymphocytic Leukemia Study Group. J Clin Oncol 30(26):3209-3216

16. Eichhorst B, Fink A-M, Bahlo J et al (2016) First-line chemoimmunotherapy with bendamustine and rituximab versus fludarabine, cyclophosphamide, and rituximab in patients with advanced chronic lymphocytic leukaemia (CLL10): an international, open-label, randomised, phase 3, non-inferiority trial. Lancet Oncol 17(7):928-942

17. Goede V, Fischer K, Busch R et al (2014) Obinutuzumab plus chlorambucil in patients with CLL and coexisting conditions. N Engl J Med 370:1101-1110

18. Hillmen P, Robak T, Janssens A et al (2015) Chlorambucil plus ofatumumab versus chlorambucil alone in previously untreated patients with chronic lymphocytic leukaemia (COMPLEMENT 1): a randomised, multicentre, open-label phase 3 trial. Lancet 385(9980):1873-1883

19. Robak T, Warzocha K, Babu G et al (2016) Ofatumumab plus fludarabine and cyclophosphamide in relapsed chronic lymphocytic leukemia: results from the COMPLEMENT 2 trial. Leuk Lymphoma 10:1-9

20. Byrd JC, Furman RR, Coutré SE et al (2013) Targeting BTK with ibrutinib in relapsed chronic lymphocytic leukemia. N Engl J Med 369:32-42

21. Oscier D, Dearden C, Eren E et al (2012) Guidelines on the diagnosis, investigation and management of chronic lymphocytic leukaemia. Br J Haematol 159:541-564

22. Dreger P, Döhner H, Ritgen M et al (2010) Allogeneic stem cell transplantation provides durable disease control in poor-risk chronic lymphocytic leukemia: long-term clinical and MRD results of the GCLLSG CLL3X trial. Blood 116:2438-2447

23. Khouri IF, Bassett R, Poindexter N et al (2011) Nonmyeloablative allogeneic stem cell transplantation in relapsed/refractory chronic lymphocytic leukemia: long-term follow-up, prognostic factors, and effect of human leukocyte histocompatibility antigen subtype on outcome. Cancer 117:4679-4688

24. Sorror ML, Storer B, Sandmaier BM, et al. (2010) Impacts of cytogenetic abnormalities and prior alemtuzumab on outcomes of patients (pts) with high-risk chronic lymphocytic leukemia (CLL) given nonmyeloablative allogeneic hematopoietic cell transplantation (HCT). [abstract 2364] Blood 116:2364. Available at: http:// www.bloodjournal.org/content/116/21/2364. Accessed January 2, 2016.

25. Brown JR, Kim HT, Armand P et al (2013) Long-term follow-up of reduced-intensity allogeneic stem cell transplantation for chronic lymphocytic leukemia: prognostic model to predict outcome. Leukemia 27:362-369

26. Toze CL, Dalal CB, Nevill TJ et al (2012) Allogeneic haematopoietic stem cell transplantation for chronic lymphocytic leukaemia: outcome in a 20-year cohort. Br J Haematol 158:174185

27. Hebenstreit K, Iacobelli S, Leiblein S et al (2014) Low tumor burden is associated with early B-cell reconstitution and is a predictor of favorable outcome after non-myeloablative stem cell transplant for chronic lymphocytic leukemia. Leuk Lymphoma 55:1274-1280

28. Dreger P, Corradini P, Kimby E et al (2007) Indications for allogeneic stem cell transplantation in chronic lymphocytic leukemia: the EBMT transplant consensus. Leukemia 21:12-17
29. Herth I, Dietrich S, Benner A et al (2014) The impact of allogeneic stem cell transplantation on the natural course of poor-risk chronic lymphocytic leukemia as defined by the EBMT consensus criteria: a retrospective donor versus no donor comparison. Ann Oncol 25: 200-206

30. Kharfan-Dabaja MA, Kumar A, Hamadani M et al (2016) Clinical practice recommendations for use of allogeneic hematopoietic cell transplantation in chronic lymphocytic leukemia on behalf of the guidelines Committee of the American Society for blood and marrow transplantation. Biol Blood Marrow Transplant 22(12):21172125

31. Dreger P, Schetelig J, Andersen N et al (2014) Managing high-risk CLL during transition to a new treatment era: stem cell transplantation or novel agents? Blood 124:3841-3849

32. Ryan CE, Sahaf B, Logan AC, et al. (2016) Ibrutinib efficacy and tolerability in patients with relapsed chronic lymphocytic leukemia following allogeneic HCT. Blood 128:2899-2908

33. Cheson BD, Byrd JC, Rai KR et al (2012) Novel targeted agents and the need to refine clinical end points in chronic lymphocytic leukemia. J Clin Oncol 30:2820-2822

34. U.S. Food and Drug Administration. FDA News Release: FDA approves Imbruvica to treat chronic lymphocytic leukemia. Silver Spring, MD: U.S. Department of Health and Human Services; 2014. Available at: http://www.fda.gov/newsevents/newsroom/ pressannouncements/ucm385764.htm. Accessed August 29, 2016.

35. U.S. Food and Drug Administration. FDA News Release: FDA expands approved use of Imbruvica for chronic lymphocytic leukemia. Silver Spring, MD: U.S. Department of Health and Human Services; 2014. Available at: http:/www.fda.gov/newsevents/ newsroom/pressannouncements/ucm406916.htm. Accessed August 29, 2016.

36. Advani RH, Buggy JJ, Sharman JP et al (2013) Bruton tyrosine kinase inhibitor ibrutinib (PCI-32765) has significant activity in patients with relapsed/refractory B-cell malignancies. J Clin Oncol 31:88-94

37. Byrd JC, Brown JR, O'Brien S, et al; for the RESONATE Investigators (2014) Ibrutinib versus of atumumab in previously treated chronic lymphoid leukemia. N Engl J Med 371:213-223

38. Byrd JC, Furman RR, Coutré SE et al (2015) Three-year follow-up of treatment-naïve and previously treated patients with CLL and SLL receiving single-agent ibrutinib. Blood 125:2497-2506

39. Farooqui M, Valdez J, Soto S, Bray A, Tian X, Wiestner A. Atrial fibrillation in CLL/SLL patients on ibrutinib. [abstract 2933] Presented at the 57th Annual Meeting and Exposition of the American Society of Hematology; December 5-8, 2015; Orlando, USA. Available at: http://www.bloodjournal.org/content/126/23/ 2933. Accessed April 4, 2016.

40. Shanafelt TD, Chaffee KG, Call TG, et al. Atrial fibrillation in patients with chronic lymphocytic leukemia (CLL). [abstract 2950] Presented at the 57th Annual Meeting and Exposition of the American Society of Hematology; December 5-8, 2015; Orlando, USA. Available at: https://ash.confex.com/ash/2015/ webprogram/Paper82991.html. Accessed January 26, 2016. 37

41. Leong DP, Caron F, Hillis C et al (2016) The risk of atrial fibrillation with ibrutinib use: a systematic review and meta-analysis. Blood 128:138-140 38

42. Imbruvica (ibrutinib) product monograph. Janssen Inc. July 19, 2016.

43. Burger JA, Tedeschi A, Barr PM et al (2015) Ibrutinib as initial therapy for patients with chronic lymphocytic leukemia. N Engl J Med 373:2425-2437

44. Cramer P, Chanan-Khan A, Fraser G, et al. Ibrutinib combined with bendamustine/rituximab (BR) in previously treated chronic lymphocytic leukemia/small lymphocytic lymphoma (CLL/SLL): safety analysis in the HELIOS trial. [abstract 102] Presented at the 16th 
International Workshop on Chronic Lymphocytic Leukaemia; September 7-9, 2015; Sydney, Australia.

45. Lipsky AH, Farooqui MZH, Tian X et al (2015) Incidence and risk factors of bleeding-related adverse events in patients with chronic lymphocytic leukemia treated with ibrutinib. Haematologica 100: $1571-1578$

46. Sun C, Tian X, Lee YS et al (2015) Partial reconstitution of humoral immunity and fewer infections in patients with chronic lymphocytic leukemia treated with ibrutinib. Blood 126:2213-2219

47. Barrientos JC, O'Brien S, Brown JR, et al. Hematologic and immunologic function and patient well-being for the phase III RESONATE $^{\text {TM }}$ study of ibrutinib vs ofatumumab in relapsed/ refractory chronic lymphocytic leukemia/small lymphocytic lymphoma. [abstract 4696] Presented at the 56th Annual Meeting and Exposition of the American Society of Hematology; December 69, 2014; San Francisco, USA. Available at: https://ash.confex.com/ ash/2014/webprogram/Paper67774.html. Accessed February 24, 2016.

48. Rogers KA, Ruppert AS, Bingman A et al (2016) Incidence and description of autoimmune cytopenias during treatment with ibrutinib for chronic lymphocytic leukemia. Leukemia 30:346-350

49. Herman SE, Gordon AL, Wagner AJ et al (2010) Phosphatidylinositol 3-kinase- $\delta$ inhibitor CAL-101 shows promising preclinical activity in chronic lymphocytic leukemia by antagonizing intrinsic and extrinsic cellular survival signals. Blood 116:2078-2088

50. Yang Q, Modi P, Newcomb T, Quéva C, Gandhi V (2015) Idelalisib: first-in-class PI3K delta inhibitor for the treatment of chronic lymphocytic leukemia, small lymphocytic leukemia, and follicular lymphoma. Clin Cancer Res 21:1537-1542

51. U.S. Food and Drug Administration. FDA News Release: FDA approves Zydelig for three types of blood cancers. Silver Spring, MD: U.S. Department of Health and Human Services; 2014. Available at: http://www.fda.gov/NewsEvents/Newsroom/ PressAnnouncements/ucm406387.htm. Accessed Sept 9, 2016.

52. Furman RR, Sharman JP, Coutré SE et al (2014) Idelalisib and rituximab in relapsed chronic lymphocytic leukemia. N Engl J Med 370:997-1007

53. Sharman JP, Coutré SE, Furman RR, et al. (2014) Second interim analysis of a phase 3 study of idelalisib (ZYDELIG®) plus rituximab (R) for relapsed chronic lymphocytic leukemia (CLL): efficacy analysis in patient subpopulations with del (17p) and other adverse prognostic factors. [abstract 330] Blood 124:330. Available at: http://www.bloodjournal.org/content/124/21/330. Accessed December 14, 2015

54. O'Brien SM, Lamanna N, Kipps TJ et al (2015) A phase 2 study of idelalisib plus rituximab in treatment-naïve older patients with chronic lymphocytic leukemia. Blood 126:2686-2694

55. Louie CY, DiMaio MA, Matsukuma KE, Coutre SE, Berry GJ, Longacre TA (2015) Idelalisib-associated enterocolitis: clinicopathologic features and distinction from other enterocolitides. Am J Surg Pathol 39:1653-1660

56. Coutré SE, Barrientos JC, Brown JR et al (2015) Management of adverse events associated with idelalisib treatment: expert panel opinion. Leuk Lymphoma 56:2779-2786

57. Gilead Sciences Inc. Decreased overall survival and increased risk of serious infections in patients receiving ZYDELIG (idelalisib). March 21, 2016. Available at: http://zydelig.com/Content/pdf/ Zydelig-Safety-Info-FINAL.pdf. Accessed April 4, 2016.

58. Anderson MA, Deng J, Seymour JF et al (2016) The BCL2 selective inhibitor venetoclax induces rapid onset apoptosis of CLL cells in patients via a TP53-independent mechanism. Blood 127:32153224

59. U.S. Food and Drug Administration. FDA News Release: FDA approves new drug for chronic lymphocytic leukemia in patients with a specific chromosomal abnormality. Silver Spring, MD: U.S.
Department of Health and Human Services; 2016. Available at: http:/www.fda.gov/newsevents/newsroom/pressannouncements/ ucm495253.htm. Accessed Sept 9, 2016.

60. Seymour JF, Davids MS, Pagel JM, et al. (2013) Bcl-2 inhibitor ABT-199 (GDC-0199) monotherapy shows anti-tumor activity including complete remissions in high-risk relapsed/refractory $(\mathrm{R} / \mathrm{R})$ chronic lymphocytic leukemia (CLL) and small lymphocytic lymphoma (SLL). [abstract 872] Blood 122:872. Available at: http:// www.bloodjournal.org/content/122/21/872. Accessed December 14, 2015.

61. Roberts A, Ma S, Brander D, et al. Venetoclax (ABT-199/GDC0199) combined with rituximab induces deep responses in patients with relapsed/refractory chronic lymphocytic leukemia. [abstract S431] Presented at the 20th Congress of the European Hematology Association; June 11-14, 2015; Vienna, Austria. Available at: http://learningcenter.ehaweb.org/eha/2015/20th/ 103125/. Accessed April 4, 2016.

62. Roberts AW, Davids MS, Pagel JM et al (2016) Targeting BCL2 with venetoclax in relapsed chronic lymphocytic leukemia. N Engl J Med 374:311-322

63. Stilgenbauer S, Eichhorst B, Schetelig J et al (2016) Venetoclax in relapsed or refractory chronic lymphocytic leukaemia with $17 \mathrm{p}$ deletion: a multicentre, open-label, phase 2 study. Lancet Oncol 17: 768-778

64. Fraser G, Cramer P, Demirkan F, et al. Ibrutinib plus bendamustine and rituximab in previously treated chronic lymphocytic leukemia/ small lymphocytic leukemia (CLL/SLL): 2-year follow-up including MRD from the HELIOS study. [abstract S430] Presented at the 21 st Congress of the European Hematology Association; June 912, 2016; Copenhagen, Denmark. Available at: http:// learningcenter.ehaweb.org/eha/2016/21st/135186/. Accessed July 19, 2016.

65. Sorenson $\mathrm{S}$, Tam $\mathrm{C}$, Diels $\mathrm{J}$ et al Indirect comparison of ibrutinib versus idelalisib plus ofatumumab in patients with previously treated chronic lymphocytic leukemia [poster]. XVI International Workshop on Chronic Lymphocytic Leukaemia; September 7-9, 2015; Sydney. Australia

66. Maddocks KJ, Ruppert AS, Lozanski G et al (2015) Etiology of ibrutinib therapy discontinuation and outcomes in patients with chronic lymphocytic leukemia. JAMA Oncol 1:80-87

67. O'Brien SM, Byrd JC, Hillmenn P, et al. Outcomes with ibrutinib by line of therapy in patients with CLL: analyses from phase III data. [abstract 7520] Presented at the 2016 Annual Meeting of the American Society of Clinical Oncology; June 3-7, 2016; Chicago, USA. Available at: http://meetinglibrary.asco.org/content/164543176. Accessed August 29, 2016.

68. O'Brien S, Furman RR, Coutre SE et al (2014) Ibrutinib as initial therapy for elderly patients with chronic lymphocytic leukaemia or small lymphocytic lymphoma: an open-label, multicentre, phase 1b/2 trial. Lancet Oncol 15:48-58

69. Woyach JA, Furman RR, Liu TM et al (2014) Resistance mechanisms for the Bruton's tyrosine kinase inhibitor ibrutinib. N Engl J Med 370:2286-2294

70. Mato A, Nabhan C, Barr PM, et al. Favorable outcomes in CLL pts with alternate kinase inhibitors following ibrutinib or idelalisib discontinuation: results from a large multi-center study. [abstract 719] Presented at the 57th Annual Meeting and Exposition of the American Society of Hematology; December 5-8, 2015; Orlando, USA. Available at: https://ash.confex.com/ash/2015/webprogram/ Paper80707.html. Accessed January 26, 2016.

71. Jones J, Mato AR, Coutre S, et al. Preliminary results of a phase 2, open-label study of venetoclax (ABT-199/GDC-0199) monotherapy in patients with chronic lymphocytic leukemia relapsed after or refractory to ibrutinib or idelalisib therapy. [abstract 715] Presented at the 57th Annual Meeting and Exposition of the American Society of Hematology; December 5-8, 2015; Orlando, USA. Available at: 
https://ash.confex.com/ash/2015/webprogram/Paper81347.html. Accessed January 1, 2016.

72. U.S. Food and Drug Administration. FDA statement: FDA halts clinical trial of drug Revlimid (lenalidomide) for chronic lymphocytic leukemia due to safety concerns. Silver Spring, MD: U.S. Department of Health and Human Services; 2013. Available at: http://www.fda.gov/Drugs/DrugSafety/ucm361444.htm. Accessed January 2, 2016.

73. Byrd JC, Harrington B, O'Brien S et al (2016) Acalabrutinib (ACP196) in relapsed chronic lymphocytic leukemia. N Engl J Med 374: 323-332

74. Chanan-Khan A, Cramer P, Demirkan F et al (2016) Ibrutinib combined with bendamustine and rituximab compared with placebo, bendamustine, and rituximab for previously treated chronic lymphocytic leukaemia or small lymphocytic lymphoma (HELIOS): a randomised, double-blind, phase 3 study. Lancet Oncol 17:200-211

75. Zelenetz AD, Robak $\mathrm{T}$, Coiffier B, et al. Idelalisib plus bendamustine and rituximab (BR) is superior to BR alone in patients with relapsed/refractory chronic lymphocytic leukemia: results of a phase 3 randomized double-blind placebo-controlled study. [abstract LBA-5] Presented at the 57th Annual Meeting and Exposition of the American Society of Hematology; December 5-8, 2015; Orlando, USA. Available at: https://ash.
confex.com/ash/2015/webprogram/Paper87420.html. Accessed February 24, 2016.

76. Jones JA, Robak T, Wach M, et al. Updated results of a phase III randomized, controlled study of idelalisib in combination with ofatumumab for previously treated chronic lymphocytic leukemia (CLL). [abstract 7515] Presented at the 2016 Annual Meeting of the American Society of Clinical Oncology; June 3-7, 2016; Chicago, USA. Available at: http://meetinglibrary.asco.org/content/163777176. Accessed November 16, 2016.

77. Barr PM, Saylors GB, Spurgeon SE et al (2016) Phase 2 study of idelalisib and entospletinib: pneumonitis limits combination therapy in relapsed refractory CLL and NHL. Blood 127:2411-2415

78. Davids MS, Kim HT, Bsat J, et al. Preliminary results of a phase II study of ibrutinib in combination with FCR (iFCR) in previously untreated, younger patients with CLL. [abstract P210] Presented at the 21st Congress of the European Hematology Association; June 9-12, 2016; Copenhagen, Denmark. Available at: http:// learningcenter.ehaweb.org/eha/2016/21st/133198/. Accessed August 29, 2016.

79. Oppermann S, Ylanko J, Shi Y et al (2016) High-content screening identifies kinase inhibitors that overcome venetoclax resistance in activated CLL cells. Blood 128:934-947 